\title{
Untreated latent tuberculosis leads to CNS lesions
}

\author{
Niki Khandheria, Roger Smalligan MD, MPH
}

\section{CASE}

A spry 85-year-old white man with a past medical history of hypertension and coronary artery disease was camping with friends when suddenly he could not speak, his face drooped to the left, and he started jerking. He had had no recent illness, no fever, no trauma, and no weight loss. The initial differential diagnosis was largely between stroke and seizure; computed tomography (CT) of the head showed a right anterior cerebral artery infarct or possible neoplasm. Magnetic resonance imaging showed four ring enhancing lesions in the right frontal cortex with vasogenic edema (Figure 1). Because of his age, metastatic disease was suspected, but CT scans of the chest and abdomen, colonoscopy, and serologic work up were all negative. Neurosurgery was consulted, and a parietal lesion was excised. Pathology showed non-caseating granulomas and rare acid fast bacilli (Figure 2). The diagnosis of central nervous system (CNS) mycobacterial disease, or tuberculoma, was made, and the patient was started on isoniazid, rifampin, pyrazinamide, and moxifloxacin. He tolerated the medications well initially but developed sepsis due to urinary tract infection and aspiration pneumonia and later died.

\section{Discussion}

Although tuberculosis (TB) is largely considered a disease of developing countries, there were 9421 new cases of TB in the USA in $2014 .{ }^{1}$ This case is unusual since only $1 \%$ of these cases involved the CNS. ${ }^{2}$ Tuberculomas more typically present in children or young adults with seizures or headache without systemic symptoms. Our patient had a family history of TB in his father and said he was told he had "dormant TB" but was never treated. He had no immunosuppressive diagnoses or drugs. Culture of his brain lesion revealed pansensitive Mycobacterium tuberculosis. Treatment of tuberculomas usually includes the drugs used in this case; ethambutol is excluded due to poor penetration of the CNS. Phase I of treatment involves two months of four medications followed by an extended phase II of isoniazid and rifampin for 18 months. Corticosteroids are considered if initial response is poor. In the past, the elderly patients were not offered treatment for latent TB, but this is now more standard to prevent reactivation disease. Physicians need to consider tuberculomas in the differential diagnosis of mass lesions in the elderly, since early diagnosis and treatment can reduce the morbidity and mortality in these cases. 


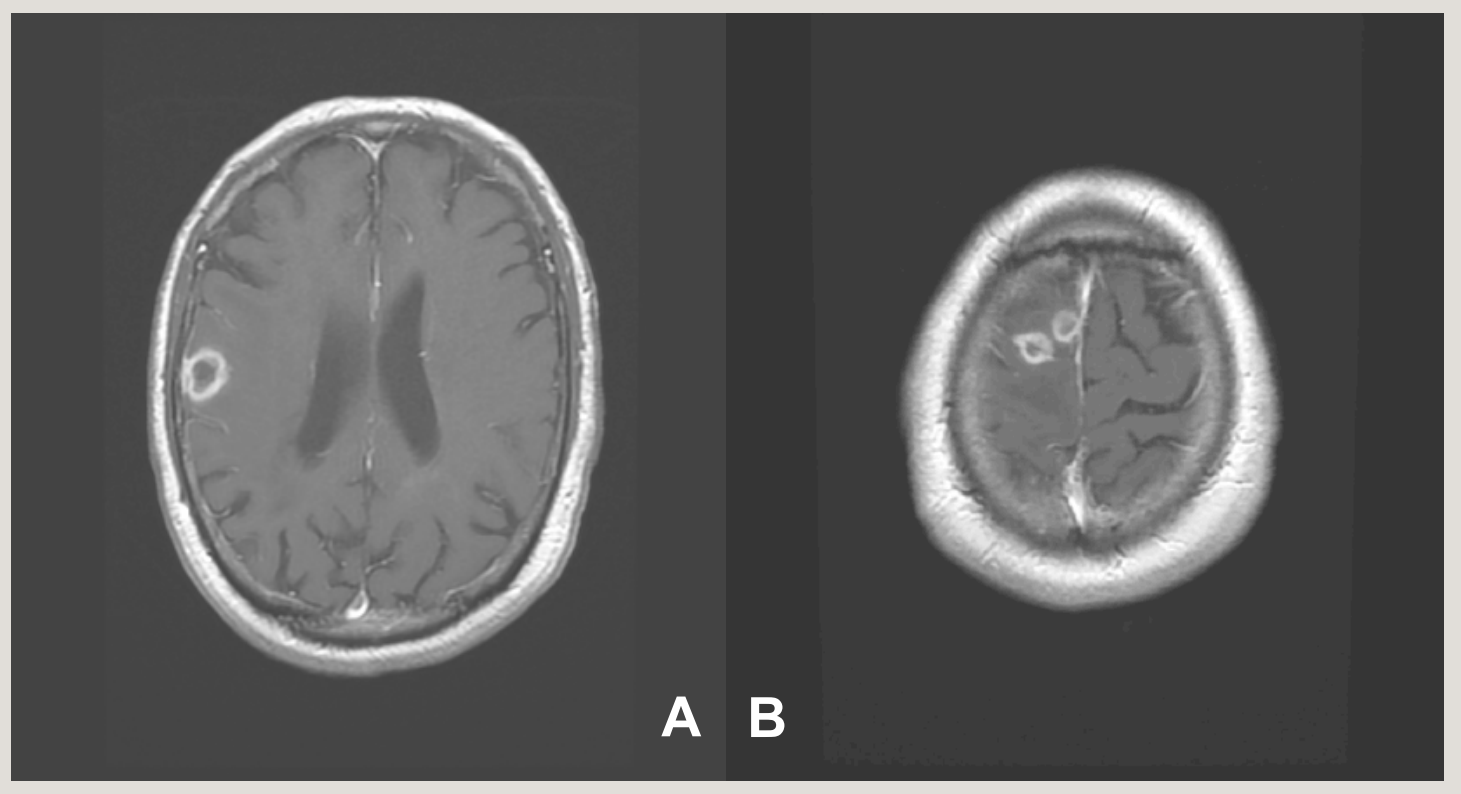

Figure $1 \mathrm{~A}$ and $\mathrm{B}$ - Contrast-enhanced axial T1W images through the brain demonstrate multiple ring-enhancing lesions in the right frontal lobe

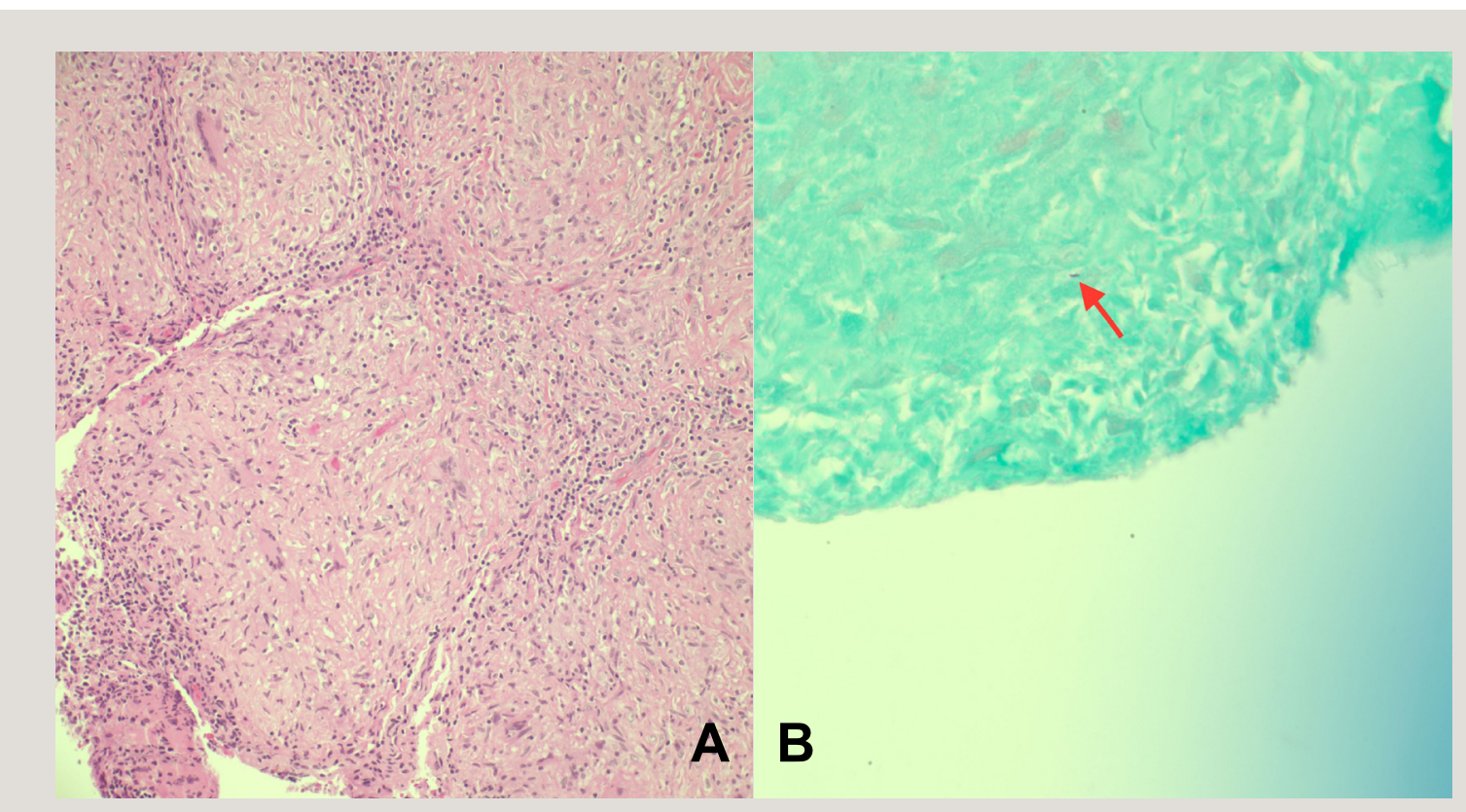

Figure 2 A and B- Non-caseating granulomatous inflammation surrounding hyalinized and focal fibrinoid necrotic tissue $(A)$; rare acid fast bacilli indicated by arrow $(B)$ 
Author Affiliation: Niki Khandheria is an undergraduate student at Georgetown University. Roger Smalligan is the chairman of medicine at Texas Tech University Health Sciences Center in Amarillo, TX.

Received: $3 / 29 / 2016$

Accepted: 6/08/2016

Reviewers: Kenneth Nugent MD

Published electronically: 07/15/2016

Conflict of Interest Disclosures: None

\section{REFERENCES}

1. Salinas JL, Mindra G, Haddad MB, Pratt R, Price SF, Langer AJ. Leveling of tuberculosis incidence - United States, 2013-2015. MMWR Morb Mortal Wkly Rep 2016; 65: 273-278.

2. Rock BR. Central nervous system tuberculosis: pathogenesis and clinical aspects. Clin Microbiol Rev 2008; 21(2): 243-261. 